SUPPORTING INFORMATION FOR:

\title{
Identification of mitosis-specific phosphorylation in mitotic chromosome-associated proteins
}

Shinya Ohta ${ }^{1 \S \star}$, Michiko Kimura ${ }^{2 \S}$, Shunsuke Takagi ${ }^{2}$, lyo Toramoto ${ }^{1}$, and Yasushi Ishihama ${ }^{2 *}$

${ }^{1}$ Center for Innovative and Translational Medicine

Medical School, Kochi University

Kohasu, Oko-cho, Nankoku, Kochi 783-8505, Japan

${ }^{2}$ Graduate School of Pharmaceutical Sciences, Kyoto University

46-29 Yoshidashimoadachi-cho, Sakyo-ku, Kyoto 606-8501, Japan

$\S$ These authors equally contributed to this work.

${ }^{*}$ Co-corresponding authors 
Table S1 Concentration of phosphopeptides

\begin{tabular}{|c|c|c|c|}
\hline Experiment & phosphopeptide & non-phosphopeptide & $\%$ of phosphopeptide \\
\hline Exp. 1-1 & 3160 & 275 & $92.0 \%$ \\
\hline Exp. 1-2 & 2117 & 293 & $87.8 \%$ \\
\hline Exp. 1-3 & 2207 & 174 & $92.7 \%$ \\
\hline Exp. 1-4 & 2116 & 250 & $89.4 \%$ \\
\hline Exp. 2-1 & 479 & 18 & $96.4 \%$ \\
\hline Exp. 2-1 & 971 & 133 & $88.0 \%$ \\
\hline Exp. 2-2 & 3025 & 242 & $92.6 \%$ \\
\hline Exp. 2-3 & 1486 & 107 & $93.3 \%$ \\
\hline Exp. 3-1 & 1754 & 116 & $93.8 \%$ \\
\hline Exp. 3-2 & 1948 & 134 & $93.6 \%$ \\
\hline Exp. 4 & 2523 & 1015 & $71.3 \%$ \\
\hline Total & 6625 & 1374 & $82.8 \%$ \\
\hline
\end{tabular}


Table S2 The detail of pie-charts in Figure 1D, 1E, and 2B

\begin{tabular}{|c|c|c|c|c|}
\hline Sub-classes & Category & $\begin{array}{l}\text { Identified } \\
\text { proteins } \\
\text { (Fig. 1D) } \\
\end{array}$ & \begin{tabular}{|c} 
Phosphorylation \\
sites \\
(Fig. 1E)
\end{tabular} & $\begin{array}{l}\text { Quantification } \\
\text { (Fig. 2B) }\end{array}$ \\
\hline Centromere & Centromere proteins & 43 & 207 & $5.57 \%$ \\
\hline \multirow{10}{*}{$\begin{array}{c}\text { Other } \\
\text { chromosomal }\end{array}$} & Telomere proteins & 7 & 51 & $0.65 \%$ \\
\hline & Chromosomal periphery proteins & 8 & 54 & $2.48 \%$ \\
\hline & Chromosomal proteins & 43 & 375 & $31.98 \%$ \\
\hline & Histones & 7 & 16 & $0.08 \%$ \\
\hline & histone modification factors & 18 & 132 & $3.89 \%$ \\
\hline & Cell-cycle proteins & 36 & 145 & $3.51 \%$ \\
\hline & Other kinases/phosphatases & 5 & 9 & $0.01 \%$ \\
\hline & Chromatin and related factors & 63 & 382 & $6.77 \%$ \\
\hline & DNA replication proteins & 22 & 66 & $9.66 \%$ \\
\hline & DNA repair proteins & 26 & 67 & $0.82 \%$ \\
\hline \multirow{11}{*}{$\begin{array}{c}\text { Nuclear and } \\
\text { RNA metabolizm }\end{array}$} & Nuclear & 77 & 349 & $0.27 \%$ \\
\hline & Transcription factors & 139 & 548 & $4.94 \%$ \\
\hline & RNA polymerases & 11 & 49 & $0.25 \%$ \\
\hline & RNA modifying enzyme and related & 16 & 48 & $0.81 \%$ \\
\hline & RNA binding proteins & 19 & 45 & $0.28 \%$ \\
\hline & RNA helicases & 6 & 31 & $0.43 \%$ \\
\hline & hnRNP & 6 & 35 & $0.04 \%$ \\
\hline & snRNP & 9 & 27 & $1.30 \%$ \\
\hline & Splicing related factors & 33 & 102 & $1.19 \%$ \\
\hline & Other translation factors & 5 & 9 & $0.01 \%$ \\
\hline & tRNA Synthetases & 1 & 1 & $0.00 \%$ \\
\hline Ribosomal & Ribosomal protein & 27 & 70 & $5.94 \%$ \\
\hline $\begin{array}{c}\text { Spindle and } \\
\text { Centrosomals }\end{array}$ & Spindle and Centrosomals & 28 & 162 & $1.66 \%$ \\
\hline \multirow{8}{*}{$\begin{array}{l}\text { Other potential } \\
\text { contaminant }\end{array}$} & Cytoskeleton proteins & 26 & 93 & $1.73 \%$ \\
\hline & Cytoplasmic proteins & 61 & 180 & $1.51 \%$ \\
\hline & Mitochondrial proteins & 12 & 35 & $0.26 \%$ \\
\hline & Membrane proteins & 33 & 78 & $0.19 \%$ \\
\hline & Receptor proteins & 26 & 118 & $3.99 \%$ \\
\hline & Chaperones & 8 & 12 & $0.26 \%$ \\
\hline & Ubiquitin related proteins & 25 & 72 & $0.36 \%$ \\
\hline & Other potential contaminant & 15 & 70 & $0.96 \%$ \\
\hline \multirow{2}{*}{ Uncharacterized } & Uncharacterized proteins & 102 & 436 & $3.77 \%$ \\
\hline & Unknown novel proteins & 52 & 203 & $4.43 \%$ \\
\hline
\end{tabular}


Table S3 Phosphorylation of Centromere proteins

\begin{tabular}{|c|c|c|c|c|c|}
\hline $\begin{array}{c}\text { Sub } \\
\text {-domain }\end{array}$ & Protein & AccNum & $\begin{array}{l}\text { phospho-site } \\
\text { in this study }\end{array}$ & $\begin{array}{c}\text { Site in Human } \\
\text { homologue }\end{array}$ & Comments \\
\hline \multirow{42}{*}{ CPC } & \multirow{2}{*}{ AuroraB } & \multirow{2}{*}{ E00001 } & T350 & L340 & \\
\hline & & & S353 & V343 & \\
\hline & \multirow{6}{*}{ Borealin } & \multirow{6}{*}{ P86346 } & S18 & A22 & \\
\hline & & & $\mathrm{S} 138$ & - & \\
\hline & & & $\mathbf{S 1 8 4}$ & S165 & Functional phosphorylation $^{1}$ \\
\hline & & & S187 & N168 & \\
\hline & & & T224 & T204 & Functional phosphorylation ${ }^{2}$ \\
\hline & & & S264 & S244 & 3,4 \\
\hline & \multirow{34}{*}{ INCENP } & \multirow{34}{*}{ F1NSZ0 } & S46 & $\mathrm{R} 47$ & \\
\hline & & & S71 & S67 & \\
\hline & & & S77 & N78 & \\
\hline & & & S122 & - & \\
\hline & & & S166 & N178 & \\
\hline & & & S183 & S208 & 5 \\
\hline & & & S185 & G210 & \\
\hline & & & S194 & T219 & 5,6 \\
\hline & & & S203 & L228 & \\
\hline & & & T217 & D242 & \\
\hline & & & S256 & S269 & 5,7 \\
\hline & & & S277 & N289 & \\
\hline & & & T280 & T292 & 8 \\
\hline & & & S294 & S306 & $4,9,10$ \\
\hline & & & S339 & N364 & \\
\hline & & & S342 & V367 & \\
\hline & & & S343 & P368 & \\
\hline & & & S348 & S373 & 7 \\
\hline & & & S350 & Q375 & \\
\hline & & & T359 & P384 & \\
\hline & & & T371 & N395 & \\
\hline & & & S399 & G428 & \\
\hline & & & S443 & Q472 & \\
\hline & & & T449 & T478 & $\begin{array}{ll}6,7,9 & 3 \\
\end{array}$ \\
\hline & & & S451 & S480 & 3 \\
\hline & & & S755 & S798 & $6,7,9,11$ \\
\hline & & & S787 & S828 & \\
\hline & & & $\mathbf{S 7 9 0}$ & S830 & \\
\hline & & & T791 & T831 & \\
\hline & & & S833 & P874 & \\
\hline & & & T851 & T892 & Functional phosphorylation by AuroraB $^{12}$ \\
\hline & & & S852 & S893 & Functional phosphorylation by AuroraB ${ }^{12,13}$ \\
\hline & & & S853 & S894 & Functional phosphorylation by AuroraB $\mathrm{B}^{12-14}$ \\
\hline & & & S858 & S899 & Functional phosphorylation by AuroraB ${ }^{15}$ \\
\hline & \multirow{7}{*}{ Sgo1 } & \multirow{7}{*}{$\mathrm{E} 1 \mathrm{C} 2 \mathrm{~W} 6$} & T432 & - & \\
\hline & & & S435 & S371 & \\
\hline & & & T437 & G373 & \\
\hline & & & S441 & D377 & \\
\hline & & & S539 & S468 & 6,7 \\
\hline & & & S541 & A470 & \\
\hline & & & S578 & S507 & Functional phosphorylation by NEK2A ${ }^{16}$ \\
\hline & HP1 & O93481 & S167 & $\mathbf{S 1 7 6}$ & 11 \\
\hline \multirow{4}{*}{ CCAN } & \multirow{4}{*}{ CENP-C } & \multirow{4}{*}{ F1NEH6 } & Y34 & 137 & \\
\hline & & & S50 & T53 & \\
\hline & & & S68 & P83 & \\
\hline & & & T69 & K84 & \\
\hline
\end{tabular}




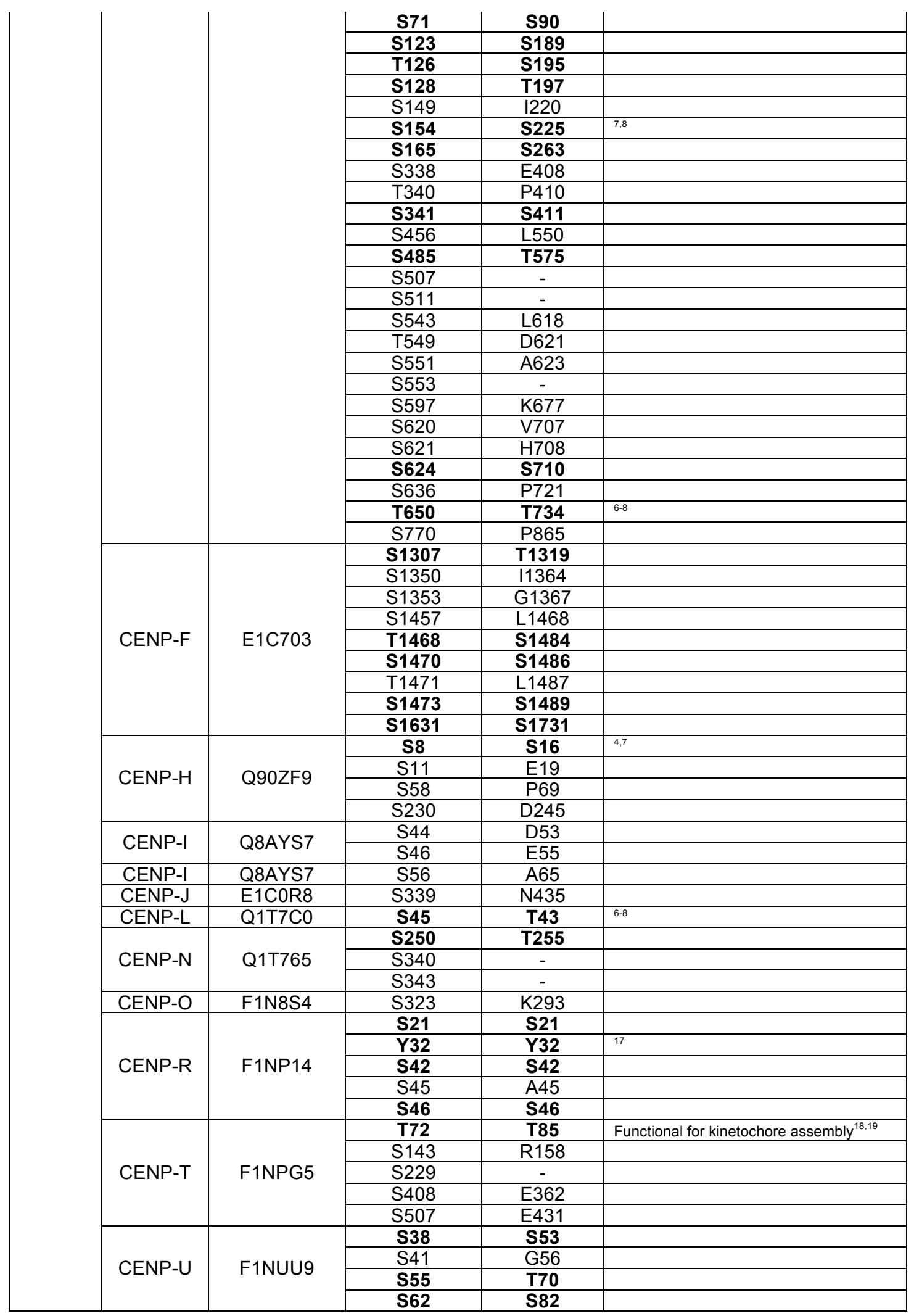




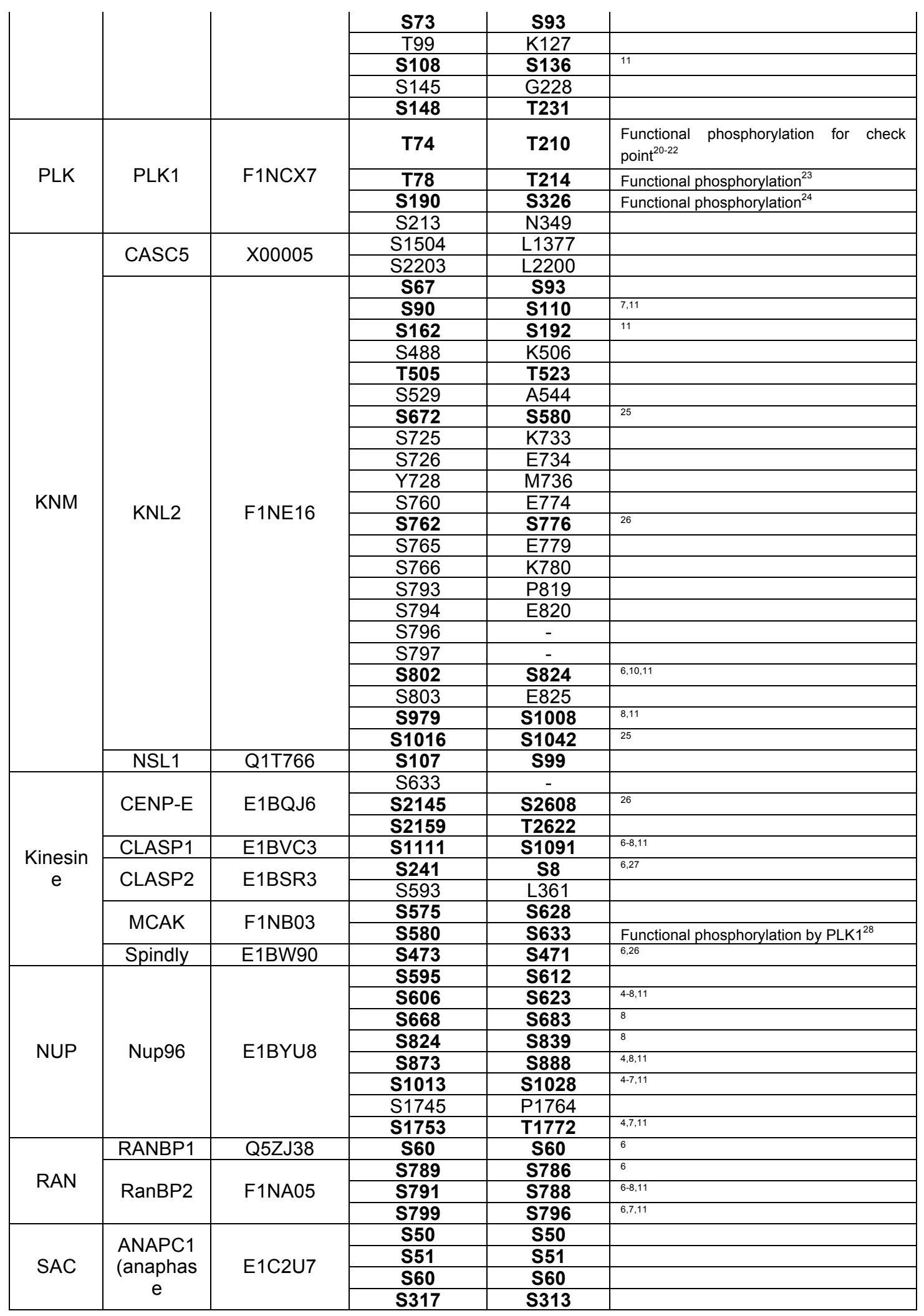




\begin{tabular}{|c|c|c|c|c|}
\hline \multirow{7}{*}{$\begin{array}{c}\text { promotin } \\
\mathrm{g} \\
\text { complex } \\
\text { subunit 1) }\end{array}$} & & S347 & S343 & \\
\hline & & S357 & A353 & \\
\hline & & S359 & S355 & \\
\hline & & S381 & S377 & \\
\hline & & S560 & S555 & \\
\hline & & S691 & S686 & \\
\hline & & S693 & S688 & \\
\hline ANAPC2 & F1NLT0 & S468 & S470 & \\
\hline \multirow{4}{*}{$\begin{array}{c}\text { APC3/CD } \\
\text { C27 }\end{array}$} & \multirow{4}{*}{ Q5ZK91 } & S268 & S267 & \\
\hline & & S277 & S276 & 5 \\
\hline & & S366 & S364 & 5 \\
\hline & & S428 & S426 & $5,6,6,8,9,11$ \\
\hline APC5 & F1NK93 & S232 & T232 & 5 \\
\hline \multirow{3}{*}{$\begin{array}{c}\text { APC6/CD } \\
\text { C16 }\end{array}$} & \multirow{3}{*}{ F1P4A5 } & S579 & T578 & \\
\hline & & S580 & G579 & \\
\hline & & T583 & P582 & \\
\hline \multirow{2}{*}{$\begin{array}{c}\text { APC8/CD } \\
\text { C23 } \\
\end{array}$} & \multirow{2}{*}{ F1NQ36 } & S585 & S588 & \\
\hline & & T593 & T596 & \\
\hline \multirow{2}{*}{ BUBR1 } & \multirow{2}{*}{ F1NYN1 } & S510 & Q516 & \\
\hline & & S512 & - & \\
\hline
\end{tabular}




\section{References}

(1) Kaur, H.; Stiff, A. C.; Date, D. A.; Taylor, W. R. Analysis of mitotic phosphorylation of borealin. BMC Cell Biol. 2007, 8 (1), 5.

(2) Kaur, H.; Bekier, M. E.; Taylor, W. R. Regulation of Borealin by phosphorylation at serine 219. J. Cell. Biochem. 2010, $111(5), 1291-1298$.

(3) Daub, H.; Olsen, J. V.; Bairlein, M.; Gnad, F.; Oppermann, F. S.; Körner, R.; Greff, Z.; Kéri, G.; Stemmann, O.; Mann, M. Kinase-selective enrichment enables quantitative phosphoproteomics of the kinome across the cell cycle. Mol. Cell 2008, 31 (3), 438-448.

(4) Malik, R.; Lenobel, R.; Santamaria, A.; Ries, A.; Nigg, E. A.; Körner, R. Quantitative analysis of the human spindle phosphoproteome at distinct mitotic stages. J. Proteome Res. 2009, 8 (10), 45534563.

(5) Hegemann, B.; Hutchins, J. R. A.; Hudecz, O.; Novatchkova, M.; Rameseder, J.; Sykora, M. M.; Liu, S.; Mazanek, M.; Lénárt, P.; Hériché, J.-K.; et al. Systematic Phosphorylation Analysis of Human Mitotic Protein Complexes. Sci Signal 2011, 4 (198), rs12-rs12.

(6) Kettenbach, A. N.; Schweppe, D. K.; Faherty, B. K.; Pechenick, D.; Pletnev, A. A.; Gerber, S. A. Quantitative phosphoproteomics identifies substrates and functional modules of Aurora and Polo-like kinase activities in mitotic cells. Sci Signal 2011, 4 (179), rs5-rs5.

(7) Santamaria, A.; Wang, B.; Elowe, S.; Malik, R.; Zhang, F.; Bauer, M.; Schmidt, A.; Sillje, H. H. W.; Körner, R.; Nigg, E. A. The Plk1-dependent phosphoproteome of the early mitotic spindle. Mol. Cell Proteomics 2011, 10 (1), M110.004457.

(8) Olsen, J. V.; Vermeulen, M.; Santamaria, A.; Kumar, C.; Miller, M. L.; Jensen, L. J.; Gnad, F.; Cox, J.; Jensen, T. S.; Nigg, E. A.; et al. Quantitative Phosphoproteomics Reveals Widespread Full Phosphorylation Site Occupancy During Mitosis. Sci Signal 2010, 3 (104), ra3-ra3.

(9) Dulla, K.; Daub, H.; Hornberger, R.; Nigg, E. A.; Körner, R. Quantitative site-specific phosphorylation dynamics of human protein kinases during mitotic progression. Mol. Cell Proteomics 2010, 9 (6), $1167-1181$.

(10) Nousiainen, M.; Sillje, H. H. W.; Sauer, G.; Nigg, E. A.; Körner, R. Phosphoproteome analysis of the human mitotic spindle. PNAS 2006, 103 (14), 5391-5396. 
(11) Dephoure, N.; Zhou, C.; Villén, J.; Beausoleil, S. A.; Bakalarski, C. E.; Elledge, S. J.; Gygi, S. P. A quantitative atlas of mitotic phosphorylation. Proc Natl Acad Sci U S A 2008, 105 (31), 10762-10767.

(12) Honda, R.; Körner, R.; Nigg, E. A. Exploring the functional interactions between Aurora B, INCENP, and survivin in mitosis. Mol. Biol. Cell 2003, 14 (8), 3325-3341. Meppelink, A.; Kabeche, L.; Vromans, M. J. M.; Compton, D. A.; Lens, S. M. A. Shugoshin-1 balances Aurora B kinase activity via PP2A to promote chromosome bi-orientation. Cell Rep 2015, 11 (4), 508515.

(14) Petsalaki, E.; Akoumianaki, T.; Black, E. J.; Gillespie, D. A. F.; Zachos, G. Phosphorylation at serine 331 is required for Aurora B activation. J. Cell Biol. 2011, 195 (3), 449-466. Hengeveld, R. C. C.; Hertz, N. T.; Vromans, M. J. M.; Zhang, C.; Burlingame, A. L.; Shokat, K. M.; Lens, S. M. A. Development of a chemical genetic approach for human aurora B kinase identifies novel substrates of the chromosomal passenger complex. Mol. Cell Proteomics 2012, 11 (5), 47-59.

(16) Fu, G.; Ding, X.; Yuan, K.; Aikhionbare, F.; Yao, J.; Cai, X.; Jiang, K.; Yao, X. Phosphorylation of human Sgo1 by NEK2A is essential for chromosome congression in mitosis. Cell Res. 2007, 17 (7), $608-618$.

(17) Zhou, H.; Di Palma, S.; Preisinger, C.; Peng, M.; Polat, A. N.; Heck, A. J. R.; Mohammed, S. Toward a comprehensive characterization of a human cancer cell phosphoproteome. J. Proteome Res. 2013, $12(1), 260-271$.

(18) Rago, F.; Gascoigne, K. E.; Cheeseman, I. M. Distinct organization and regulation of the outer kinetochore KMN network downstream of CENP-C and CENP-T. Curr. Biol. 2015, 25 (5), 671-677.

(19) Nishino, T.; Rago, F.; Hori, T.; Tomii, K.; Cheeseman, I. M.; Fukagawa, T. CENP-T provides a structural platform for outer kinetochore assembly. EMBO J. 2013, 32 (3), 424-436.

(20) Macůrek, L.; Lindqvist, A.; Lim, D.; Lampson, M. A.; Klompmaker, R.; Freire, R.; Clouin, C.; Taylor, S. S.; Yaffe, M. B.; Medema, R. H. Polo-like kinase-1 is activated by aurora A to promote checkpoint recovery. Nature 2008, 455 (7209), 119-123.

(21) van de Weerdt, B. C. M.; van Vugt, M. A. T. M.; Lindon, C.; Kauw, J. J. W.; Rozendaal, M. J.; Klompmaker, R.; Wolthuis, R. M. F.; Medema, R. H. Uncoupling anaphase-promoting complex/cyclosome activity from spindle assembly checkpoint control by deregulating polo-like kinase 1. Mol. Cell. Biol. 2005, 25 (5), 2031-2044. 
(22) Smits, V. A.; Klompmaker, R.; Arnaud, L.; Rijksen, G.; Nigg, E. A.; Medema, R. H. Polo-like kinase-1 is a target of the DNA damage checkpoint. Nat Cell Biol 2000, 2 (9), 672-676.

(23) Lasek, A. L.; McPherson, B. M.; Trueman, N. G.; Burkard, M. E. The Functional Significance of Posttranslational Modifications on Polo-Like Kinase 1 Revealed by Chemical Genetic Complementation. PLOS ONE 2016, 11 (2), e0150225.

(24) Tang, J.; Yang, X.; Liu, X. Phosphorylation of Plk1 at Ser326 regulates its functions during mitotic progression. Oncogene 2008, 27 (52), 6635-6645.

(25) Mertins, P.; Qiao, J. W.; Patel, J.; Udeshi, N. D.; Clauser, K. R.; Mani, D. R.; Burgess, M. W.; Gillette, M. A.; Jaffe, J. D.; Carr, S. A. Integrated proteomic analysis of post-translational modifications by serial enrichment. Nat. Methods 2013, 10 (7), 634-637.

(26) Sharma, K.; D'Souza, R. C. J.; Tyanova, S.; Schaab, C.; Wiśniewski, J. R.; Cox, J.; Mann, M. Ultradeep human phosphoproteome reveals a distinct regulatory nature of Tyr and Ser/Thr-based signaling. Cell Rep 2014, 8 (5), 1583-1594.

(27) Christensen, G. L.; Kelstrup, C. D.; Lyngsø, C.; Sarwar, U.; Bøgebo, R.; Sheikh, S. P.; Gammeltoft, S.; Olsen, J. V.; Hansen, J. L. Quantitative phosphoproteomics dissection of seven-transmembrane receptor signaling using full and biased agonists. Mol. Cell Proteomics 2010, 9 (7), 1540-1553.

(28) Ritter, A.; Sanhaji, M.; Steinhäuser, K.; Roth, S.; Louwen, F.; Yuan, J. The activity regulation of the mitotic centromere-associated kinesin by Polo-like kinase 1. Oncotarget 2015, 6 (9), 6641-6655. 